ORIGINAL PAPER

The Effectiveness of Acceptance and Commitment Therapy for Adolescent Mental Health: Swedish and Australian Pilot Outcomes

Fredrik Livheim Louise Hayes Ata Ghaderi Thora Magnusdottir Anna Högfeldt Julie Rowse Simone Turner Steven C. Hayes \& Anders Tengström

F. Livheim $\square$ A. Tengström

Department of Clinical Neuroscience, Karolinska Institutet, Stockholm, Sweden

Mail: Forum, Norra Stationsgatan 69, floor 7, 113 64, Stockholm, Sweden.

E-mail: fredrik.livheim@ki.se

L. Hayes

Orygen Youth Health Research Centre and

Centre for Youth Mental Health, University of Melbourne, Australia.

A. Ghaderi

Division of Psychology, Department of Clinical Neuroscience, Karolinska Institutet, Stockholm, Sweden

T. Magnusdottir A. Högfeldt

Department of Psychology, Uppsala University, Uppsala, Sweden

\title{
J. Rowse S. Turner
}

Ballarat Health Services, Victoria Australia

S. C. Hayes

Department of Psychology, University of Nevada, Reno, USA 


\begin{abstract}
Depression, anxiety and stress are common problems among adolescents. Teaching young people coping strategies in school-based intervention programs is one promising approach hoped to remedy the negative consequences of distress in adolescence. The aim of the two pilot studies was to examine the effect of a brief intervention based on the principles of Acceptance and Commitment Therapy (ACT) on depressive symptomatology (Australian study, $N=66$ ) and stress (Swedish study, $N=32$ ) among adolescents screened for psychosocial problems in school settings. In both studies, subjects were assigned to receive the ACT-groupintervention, or a control intervention featuring individual support from the school health care. The Australian study was a planned comparison, with random allocation for girls, plus one replication of a boys group. The Swedish study used a randomized controlled design. The ACT-intervention was an 8-session manualized group program. The Australian study showed significant reductions in depressive symptoms with a large effect, and significant reductions in psychological inflexibility with a medium effect when compared to the control group who received standard care. In the Swedish study, the ACT-intervention group, when compared to the control group, reported significantly lower levels of stress with a large effect size, and marginally significant decrease of anxiety, and marginally significant increased mindfulness skills. Taken together, the ACT-intervention seems to be a promising intervention for reducing stress and depressive symptoms among young adolescents in school and should be tested in full-sized studies. Limitations of these two pilots include small samples.
\end{abstract}

Keywords: Adolescent Depression, Adolescent Stress, ACT (Acceptance and Commitment Therapy), school intervention. 


\section{Introduction}

In any given year, about $20 \%$ of adolescents globally will experience a mental health problem, most commonly depression or anxiety (World Health Organization, 2011). Rates of clinical depression among adolescents is high, with $6 \%$ at the age of 15 years, followed by a steep increase to $17.4 \%$ at the age of 18 years (Merry \& Spence, 2007). Clinical depression among young people is associated with a lot of suffering. Of concern is also that depression in adolescence increases the likelihood of recurrent depression in adulthood (Keenan-Miller, Hammen, \& Brennan, 2007), with a $40 \%$ and $70 \%$ cumulative probability of re-occurrence within two and five years respectively (Parker \& Roy, 2001).

The high and increasing rates of stress and mental health conditions among adolescents' calls for more research on prevention interventions. In a Swedish study it was found that $44 \%$ of Sweden's 15 year olds (52\% of girls and $35 \%$ of the boys) report being stressed due to self-imposed demands (Statistics Sweden, 2010). Furthermore, chronic stress during adolescence is one of the most powerful predictors for the development of mood disorders, especially for adolescents who experience early-life adversity and high levels of on-going chronic stress (Grant, Compas, Thurm, McMahon, \& Gipson, 2004; Hankin, Mermelstein, \& Roesch, 2007; Rao, Hammen, Ortiz, Chen, \& Poland, 2008).

Given the high prevalence, and increase of adolescent stress and mental health conditions, it is of great importance to find effective, preventive interventions (Calear \& Christensen, 2010). Prevention programs are often divided into three different categories, - universal, selective and indicated (Mrazek \& Haggerty, 1994). Universal programs are delivered to all students regardless of symptom level. Selective programs target children and adolescents who are at risk of developing disorders. And indicated programs target individuals with mild or early symptoms (Mrazek \& Haggerty, 1994).

The first systematic Cochrane review of school-based programs for adolescent depression concluded that targeted programs were more effective than universal programs (Calear \& Christensen, 2010; Horowitz, Garber, Ciesla, Young, \& Mufson, 2007; Merry, McDowell, Hetrick, Bir, \& Muller, 2004). The second Cochrane review published 2011 (Merry et al., 2011) once again showed that targeted programs were effective, but this review also revealed stronger findings for universal programs. Earlier reviewers have commented on the larger effect size with targeted intervention rather than universal intervention (e.g. Horowitz, 2007). Because the reviews of targeted interventions include indicated interventions where young people are recruited because of elevated symptoms, a larger effect size is to be expected. In the meta-analysis of Merry et al (2011) there was evidence that both universal and targeted interventions are effective approaches. Thus there is ample need for new 
research in schools. Preventing the onset of depression and stress related suffering would be an important advance in public health (Duckworth, Kim, \& Tsukayama, 2013; Merry et al., 2011).

Another distinction that is often used in clinical research is the difference between efficacy and effectiveness trials (Flay et al., 2005). Both efficacy and effectiveness trials have been conducted in the school setting. Efficacy trials assess an intervention under optimal and controlled conditions, while effectiveness trials evaluate a program under naturalistic or real-world conditions that approximate usual care (Calear \& Christensen, 2010).

Acceptance and commitment therapy is a cognitive-behavioral approach with a firm theoretical basis, which give it the potential to work trans-diagnostically, across different contexts, and with a wide array of adolescent problems (S.C. Hayes, Strosahl, \& Wilson, 2012).

The goal of ACT is to increase psychological flexibility, which is defined as the ability to contact the present moment, and based upon what the situation affords, to change or persist in behaviour in accordance with one's values (S. C. Hayes \& Strosahl, 2004). ACT uses a unified model through six core processes: defusion, acceptance, flexible attention to the present moment, self-as-context, values, and committed action. When present, these six core processes together make up the construct psychological flexibility (see S. C. Hayes, Strosahl \& Wilson, 2012). This is relevant for prevention as it is a broad model of how to live more effectively, rather than focusing on elimination of symptoms. The effect of living a more vital life is that self-reported psychological symptoms decline (Biglan, Hayes, \& Pistorello, 2008).

Notably, fairly short ACT interventions have proven effective for a wide variety of problems among adults, like depression, stress, burnout, drug abuse, chronic pain, anxiety, coping with psychosis, smoking cessation, prejudice, deliberate self-harm, obsessive compulsive disorder and epilepsy (S. C. Hayes, Luoma, Bond, Masuda, \& Lillis, 2006). Meta-analytic studies have shown that ACT is effective with generally medium to large effect sizes when compared to wait lists or treatment as usual across a very wide range of problem areas (S. C. Hayes et al., 2006; Ost, 2008; Powers, Vording, \& Emmelkamp, 2009).

ACT has been applied across a variety of child and adolescent populations, although evidence is still preliminary. Empirical support for ACT interventions with adolescents includes: chronic pain (Wicksell, Melin, Lekander, \& Olsson, 2009), female high-risk sexual behaviour (Metzler, Biglan, Noell, Ary, \& Ochs, 2000), female anorexia (Heffner, Sperry, Eifert, \& Detweiler, 2002) and depression in psychiatric outpatient settings (L. L. Hayes, Boyd, \& Sewell, 2011). 
With regard to school settings, there have been three earlier studies, all unpublished. Of these studies, one was aimed at youth at risk (Wilson \& Murrell, 2002), a second was aimed at reducing the impact of relational aggression (Theodore-Oklota \& Orsillo, 2011), and the third was aimed at preventing stress across a two-year follow-up (Jakobsson \& Wellin, 2006; Livheim, 2004). This latter Swedish program was subsequently replicated with social workers (Brinkborg, Michanek, Hesser, \& Berglund, 2011). The Livheim et al. (2004) study was delivered as a universal prevention program with 230 students aged 16-19 years, they received 9 hours (3 sessions) of prevention and data was compared to a passive control condition. Results showed significantly improved outcomes up to two years later on measures of stress, anxiety, and psychological flexibility, all with small effect sizes. In summary, there is some evidence to support further investigation using ACT with young people in schools, however there is a lag behind adult studies making it important to pave the way for further work. Nowhere is this more important than in school settings.

The present study represents a trans-diagnostic test because it uses the same intervention for depression (Australia) and stress (Sweden). Given the pilot-nature of those trials with expected low numbers of participants, the studies were set up as targeted interventions to avoid floor effects. And to get an indication of the effectiveness of the intervention, the studies they were conducted under real-world conditions in ordinary schools.

The Australian study tested the hypothesis that a targeted ACT group intervention for adolescents would be more effective than a treatment as usual (TAU) control condition on the primary outcome variable of depression

The Swedish study was a pilot effectiveness study, testing the hypothesis that ACT as a targeted group intervention for adolescents would be more effective then treatment as usual (TAU), in the form of individual support from the school nurse (standard care for students at risk), on the primary outcome variable self-perceived stress. 


\section{Method}

\section{The Australian study}

\section{Participants}

Eligible participants were students aged 12 to 18 years attending the included schools. Initially, the school counsellor/welfare coordinators were asked to nominate students and invite them to participate if they were experiencing mild to moderate depressive symptoms including: anxious thoughts, change in appetite or weight, depressed mood, feelings of worthlessness, irritability, loss of interest, reduced ability at school, or social withdrawal. Counsellors/welfare coordinators were instructed not to nominate participants if they were experiencing severe symptoms, suicidality, or complete withdrawal from school. Those students who expressed an interest in participating were provided with a plain language statement and asked to obtain written parental consent. To confirm inclusion criteria and screen for risk, the researchers then met with each participant individually, explained the program, conducted a brief clinical interview and administered each measure. No students were excluded as a result of this interview. The number of ACT-sessions attended is not available, however, students were required to attend at least 4 sessions. Students who did not meet this criterion were considered non-completers. Sixty-six participants were enrolled in the study, with post data available from 51 adolescents aged between 12.5 and 17.75 years $(M=14.6, S D=1.03)$. Given the age of the participants, the program was conducted in single sex groups and the majority of participants were female, with only one male group conducted (Males, $\mathrm{n}=8$ ). All participants were born in Australia and the majority were living with their mother and father $(80 \%)$.

\section{Participant flow}

Please insert Figure 1. Participant Flow Diagram Australia here 
Study settings

The study took place in Australian high schools. Four of the five schools were from a regional area with a population of 100,000 while the remaining school was located in a small town with a population of 15,000 . One school was an alternative school for students who had been unable to cope with mainstream high schools and this school had notable high dropout rates $(n=7)$

\section{Ethical considerations}

This study was reviewed and formally approved of by the local Ethical Review Board in Melbourne, Victoria (Registration number: SOS003771). The study also complied with the considerations of the local Ethical Review Board and the Helsinki Declaration (World Medical Association, 2008). All participants gave their informed consent prior to their inclusion in the study. The participants were invited by their school counselor without obligation to attend. Before participant's inclusion, written parental consent was received.

\section{Interventions}

The intervention was the ACT Experiential Adolescent Group, this is a manualized 8-week group program (L. L. Hayes \& Rowse, 2008) available from the Association of Contextual Behavioral Science, www.contextualscience.org). The program uses experiential mediums, for example painting and role-play, to facilitate adolescents' experience of the six ACT processes. The use of art allows adolescents to explore their own experiences - increasing the opportunity for the processes to be new and fresh and reducing the likelihood of getting caught up in language processes. The group program was delivered to five schools, the facilitators were registered psychologists and co-facilitated by clinical psychology graduate students ( 3 schools) or the schools own counsellor ( 2 schools). All staff received a minimum 2 days training in ACT and were supervised by the authors of the program.

In this study the comparison consisted of 12-weeks of monitoring support from the school counsellor, which is the standard care provide by the school for students identified as at risk.

\section{Measures}

The primary outcome measure was improvements in depressive symptoms as measured by the RADS-2 (Reynolds Adolescent Depression Scale-2). A secondary measure was improvements in psychological 
inflexibility as measured by the AFQ-Y8 (Avoidance and Fusion Questionnaire-Youth, 8 item). Psychological inflexibility was considered to be the primary process variable

RADS-2: The Reynolds Adolescent Depression Scale-2 (RADS-2) is a brief 30 item self-report measure designed to measure depressive symptoms in adolescents (Reynolds, 2010). RADS-2 measures current levels of depressive symptomatology along four dimensions: Dysphoric Mood, Anhedonia/Negative Affect, Negative Self-Evaluation and Somatic Complaints, and also provides a Total Depression score. RADS-2 shows adequate psychometric properties (Reynolds, 2010). Internal reliability on a school sample of participants $(N=9052)$ is strong with Cronbach's alpha of .93 for the total score, and coefficients ranging from .80 to .87 for the four subscales (Reynolds, 2002). Cronbach's alpha for this sample was .87.

$S D Q$ : The Strengths and Difficulties Questionnaire (SDQ), was used to screen for clinical severity. The SDQ is a brief behavioral screening questionnaire that measures a wide range of symptoms, from emotional and conduct problems to hyperactivity and peer relationship problems (Goodman, Meltzer, \& Bailey, 2003) with good psychometric properties (Muris, Meesters, Eijkelenboom, \& Vincken, 2004). The full scale of the youth self-report version has sound reliability at $=.80$ (Goodman, 2001) and in this study Cronbach's alpha was .74.

AFQ-Y8: The Avoidance and Fusion Questionnaire for Youth, short form (Greco, Baer, \& Lambert, 2008), is an eight item self-report measure that is designed to measure psychological inflexibility. In accordance with the theoretical foundations of Acceptance and Commitment Therapy, psychological inflexibility is seen in excessive cognitive fusion (for example, 'the bad things I think about myself must be true'), and experiential avoidance. The AFQ-Y8 provides a single score with higher scores suggestive of greater inflexibility. The AFQY8 has good internal consistency with Cronbach's alpha of .83 and has been validated against established measures of psychological distress as well as ACT consistent measures (Greco et al., 2008). Internal alpha for this sample was .89 at pre-intervention.

Program Satisfaction Measure: Satisfaction with the program was measured via six qualitative questions, answered anonymously, asking the participants to report on their experience of the group. For example, participants were asked to state what they liked most about the group, and what they would like to change about the group.

Please insert Table 1 about here (Correlation matrix Australian study) 


\section{Group Allocation}

Given the pilot nature of the study, no power analysis was made. Given the median age of participants was 14-15 years, single sex groups were considered more suitable and would allow participants to fully engage with the program. Female participants were randomly allocated to the ACT groups $(n=32)$ or control group ( $n=$ 26) using a random number table with names concealed from the researchers and school personnel. The males were not randomized, and all males $(n=8)$ got the ACT-intervention in one single group, this has been addressed in the data analysis.

\section{Data Analyses}

Mixed Model Repeated Measures (MMRM) was used to investigate effects for data collected at pre and post (Verbeke \& Molenberghs, 2000). MMRM uses all available data for all participants and adjusts for missing data and patterns in the obtained data linked to drop out, and thus MMRM is well suited to intent-to-treat analyses (ITT). In the Australian study the fixed effects were; a) Intercept, b) School, c) Age, d) Time, e) Condition, and f) Condition * time. Subjects were treated as a random factor. No bootstrapping was used. Results were based on the unstructured covariance model. Denominator degrees of freedom for the fixed effects test statistics were based on the Sattherthwaite approximation. Effects sizes (Cohen's $d$ ) for $F$ values were based on the method suggested for repeated measures and multilevel designs by Rosenthal and Rosnow (1991) and for MMRM contrasts as specified by Wackerly, Mendenhall, and Scheaffer (2008).

Since the group of boys were not randomized, males were included in the primary analysis after first determining that there were not even marginally significant gender by time interactions within the experimental group (that is, the changes from pre to post was the same for females and males in the intervention group); however all analyses were supplemented by secondary analysis solely with the females, who were fully randomized. Effect sizes used the cutoffs suggested by Cohen (1988): small >0.20, medium $>0.50$, large $>0.80$. Analyses with probabilities of $p=<0.10$ but $>0.05$ were referred to as "marginally significant" and were interpreted. Significance values that exceeded $p=0.001$ are reported as $p<0.001$ regardless of value.

\section{Results}

Table 1 shows the adjusted means for all outcome measures for the two conditions for each measurement occasion drawn from mixed model analyses. The MMRM results are shown in Table 1. 
Tests of pre-differences with all participants included were drawn from contrasts conducted in MMRM analyses. At baseline, the ACT-intervention group had marginally significantly higher depression overall [RADS-2, $(t(50)=-1.82 p=0.075$, Cohen's $d=0.54]$, significantly higher levels of the dysphoric subscale [RADS-2 Dysphoric subscale $(t(28)=-2.61 p=0.012$, Cohen's $d=0.77]$. No other measures were significantly different at baseline.

Tests of pre-differences when only girls were included were also drawn from contrasts conducted with MMRM analyses. At baseline, the intervention group had significantly higher overall depression [RADS-2, $(t$ $(42)=-10.57 p=0.022$, Cohen's $d=0.72]$, significantly higher levels on the Dysphoria subscale $[t(42)=-3.16$ $\mathrm{p}=0.003$, Cohen's $d=0.96]$, marginally higher levels of Somatic Complaints $[t(44)=-1.74 \mathrm{p}=0.089$, Cohen's $d$ $=0.52]$, marginally higher levels on the Negative Self-Evaluation subscale $[t(43)=-1.82 p=0.076$, Cohen's $d=$ 0.55], and significantly higher levels of psychological inflexibility [AFQ-Y8 $(t(43)=-2.02 p=0.050$, Cohen's $d$ $=0.60]$. RADS-2 Anhedonia/Negative Affect subscale was not significantly different.

All primary analyses were supplemented with analyses using baseline covariates of age and school to adjust for these differences.

Please insert Table 2 Mean, standard deviation, and MMRM about here

Please insert Figure 2. Primary outcome Australia, Intervention Effects on depression about here

Results for the Reynolds Adolescent Depression Scale-2 (RADS-2) showed a non-significant effect for time $(p=0.728)$, a non-significant effect for condition $(p=0.664)$, and a significant and large time by treatment interaction $(F(1,44.7)=7.59, p=0.008$, Cohen's $d=0.86)$. As is shown in Figure 3, the interaction was accounted for by the marginally significant deterioration in the TAU condition $($ Mdiff $=6.95, \mathrm{SE}=3.65, t$ 
$(45.19)=1.90, p=0.064,95 \% \mathrm{CI}:-0.41,14.31)$ and the significant improvement in the ACT condition $($ Mdiff $=$ $-5.38, \mathrm{SE}=2.58, \mathrm{t}(43.79)=-2.09, p=0.043,95 \% \mathrm{CI}:-10.58,-0.18)$

Results for depressive symptomatology on the Dysphoric subscale showed a non-significant effect for time $(p=0.950)$, a non-significant effect for condition $(p=0.24)$, and a significant and medium time by treatment interactions $(F(1,45.0)=6.09, p=0.017$, Cohen's $d=0.77)$. For Anhedonia/Negative there was a non-significant effect for time $(p=0.883)$, a non-significant effect for condition $(p=0.735)$, and a significant and large time by treatment interaction $(F(1,46.2)=8.31, p=0.006$, Cohen's $d=0.89)$. For the Negative SelfEvaluation subscale there was a non-significant effect for time $(p=0.497)$, a non-significant effect for condition $(p=0.731)$, and a significant and medium time by treatment interactions $(F(1,45.5)=4.63, p=0.037$, Cohen's $d=0.67)$. For the Somatic Complaints subscale there was a non-significant effect for time $(p=0.980)$, a nonsignificant effect for condition $(p=0.773)$, and a non-significant and medium time by treatment interaction $(F$ $(1,45.1)=2.62, p=0.113$, Cohen's $d=0.50)$.

The primary process variable was considered to be levels of psychological inflexibility as measured by the Avoidance and Fusion Questionnaire short form (AFQ-Y8). Results for the AFQ-Y8 showed a nonsignificant effect for time ( $p=0.879)$, a non-significant effect for condition $(p=0.610)$, and a significant and medium time by treatment interactions $(F(1,48.34)=5.74, p=0.021$, Cohen's $d=0.73)$. The interaction was accounted for by the non-significant increase of psychological inflexibility in the TAU condition $($ Mdiff $=2.08$, $\mathrm{SE}=1.32, t(49.19)=-1.58, p=0.121,95 \% \mathrm{CI}:-0.57,4.73)$ and the marginally significant improvements in the ACT condition $($ Mdiff $=-1.83, \mathrm{SE}=0.96, t(46.76)=-1.90, p=0.063,95 \% \mathrm{CI}:-3.76,0.10)$.

As this was an exploratory program, it was important to obtain feedback from participants on the design, program content, and their perceptions of the program outcomes. Qualitative answers on the satisfaction survey were as follows:

To the question - What did you most like about the group? - the most common themes were that (1) we learned about feelings, (2) we learned everyone was the same, and (3) and it was fun. To the question - What was the most important thing you learned? - the most common themes were (1) thoughts and feelings do not need to rule my life, and (2) my feelings are normal. And to the question - Was there anything you didn't like about the group? - the majority answered 'no' with a small number saying 'it was too short' or 'it was sometimes confusing. 
In terms of acceptability, the responses were unanimously positive with the majority of participants responding 'No' to the question: 'Was there anything you did not like about this group program?' Participants reported that the group was "good fun", and the overall low dropout rate further supports this for participants who commenced the program. 


\section{The Swedish study}

\section{Participants}

Eligible participants were students aged 14-15 years in 10 classes in the participating school. Inclusion criteria were: ability and willingness to participate in the intervention program, and scoring above the $80^{\text {th }}$ percentile on the scales measuring psychological problems (see outcome measures). Exclusion criteria were: ongoing medical or psychological treatment for psychological or psychiatric problems, occurrence of suicidal thoughts or behaviour or other signs of severe psychiatric problems according to the assessments of the school health service. Participants were 32 adolescents. As with the Australia study, given the age of the participants, the program was conducted in single sex groups and the majority of participants were female $($ Males, $n=9)$.

\section{Participant flow}

Please insert Figure 3. Participant Flow Diagram Sweden here

Study settings

The study took place in Sweden. The school was a regular public high school. The Chief Physician of the schools in the county chose the school because of many reports of high levels of stress in this particular school. The only notable differences from other regular public high school is that all of the students at this school qualified to study at upper secondary school at the age of 15 compared to $89 \%$, which is the national average rate, and parent's income level was twice as high as the Swedish family mean.

\section{Ethical considerations}

This study was reviewed and formally approved of by the local Ethical Review Board in Stockholm (Registration number: 2011/5:4). The study also complied with the considerations of the local Ethical Review Board and the Helsinki Declaration (World Medical Association, 2008). All participants gave their informed consent prior to their inclusion in the study. Before participant's inclusion, written parental consent was 
received. In Sweden the intervention was intended and advertised as stress management training, this was also a step to minimize the risk of stigmatizing the participants.

\section{Interventions}

The Swedish program addressed stress more explicitly compared to the Australian study as this condition was perceived to be the major problem at the school. The Australian ACT-protocol (L. L. Hayes \& Rowse, 2008) was translated to Swedish and tested on a non-clinical group ahead of the current pilot study. Due to time constraints, the 8 group-sessions were adapted to fit within a period of 6 weeks. The ACT-sessions were given after school hours, but as close to the last lesson as possible. Each session lasted approximately 90 minutes. Two clinical psychology major students, with clinical training in CBT and four days ACT-training, delivered the group program. Both psychology students participated at all sessions.

In this study the TAU comparison consisted of individual support by the school nurse, which is the standard care provided by the school for students identified as at risk. Half of the participants in the TAU group $(n=7)$ met the school nurse for individual counselling, ranging between 2-8 sessions.

\section{Measures}

Screening measures: To identify participants that met with the inclusion criteria of scoring above the $80^{\text {th }}$ percentile on the scales measuring psychological problems, the following screening measures were used; SDQ (Strengths and Difficulties Questionnaire), PSS (The Perceived Stress Scale), and GHQ-12 (General Health Questionnaire).

SDQ: The SDQ is described in the Australian study. The SDQ has been translated into Swedish and backtranslated into English by a bi-lingual person.

GHQ-12: The General Health Questionnaire, 12 items, is the short version of the GHQ-60, a screening instrument used for detecting cases of psychological distress, targeting anxiety, depression, social dysfunction, and loss of confidence (Gao et al., 2004). GHQ-12 has been translated, and back-translated by a bi-lingual person. The GHQ-12 has good psychometric properties (Campbell, Walker, \& Farrell, 2003; Gao et al., 2004; Montazeri, Hole, Milroy, McEwen, \& Gillis, 2004; Politi, Piccinelli, \& Wilkinson, 1994). Cronbach's alpha for this sample was .81.

PSS: The Perceived Stress Scale is a self-reported instrument designed to measure the degree to which situations in one's life are appraised as stressful (S. Cohen \& Janicki-Deverts, 2012). PSS has been translated, and back-translated by a bi-lingual person. A number of studies suggest that the psychometric properties of the 
scale for evaluating perceived stress are adequate (S. Cohen \& Janicki-Deverts, 2012; S. Cohen, Kessler, \& Underwood Gordon, 1995; Hewitt, Flett, \& Mosher, 1992), including the Swedish population. For Swedish adults, low levels of stress are defined as 24 points or less, and high levels of stress defined as 25 points or more (Eskin \& Parr, 1996). Cronbach’s alpha for this sample was .76.

$D A S-S$ 21: Depression, Anxiety and Stress Scale (DAS-S) is a 21-item self-report measure designed to measure depression, anxiety and stress. DAS-S has been translated, and back-translated by a bi-lingual person. DAS-S shows adequate psychometric properties (T. A. Brown, Chorpita, Korotitsch, \& Barlow, 1997). Cronbach's alphas for the respective subscales in this sample were; stress .83 , depression .78 and anxiety .72 .

SWLS: Satisfaction with Life Scale is a 5-item self-report measure designed to measure subjective wellbeing. The participants answer questions that compare their current life situation with how they want their life to be like. SWLS has been translated, and back-translated by a bi-lingual person. SWLS shows good psychometric properties (Neto, 1993; Pavot \& Diener, 1993). Cronbach's alpha for this sample was .78.

AFQ-Y17: Avoidance and Fusion Questionnaire for Youth (Greco et al., 2008), is an ACT-specific, 17item measure generated to measure experiential avoidance and cognitive fusion in youth. The AFQ-Y can also be said to measure "psychological inflexibility" which is a construct made up of experiential avoidance and cognitive fusion. Results of classical test theory, factor analysis, and item response theory support the psychometric properties of the instrument. AFQ-Y17 has been translated, and back-translated by a bi-lingual person. The AFQ- Y17 shows good internal consistency with Cronbach's alpha of .90 (Greco et al., 2008). Cronbach's alpha for this sample was .87 .

MAAS: Mindful Attention Awareness Scale is a 15-item scale designed to assess how attentive a person is of what is taking place in the present, with higher scores indicating higher levels of mindfulness. MAAS has been translated, and back-translated by a bi-lingual person. The scale has been validated in a number of studies and shows strong psychometric properties (K. W. Brown \& Ryan, 2003). Cronbach's alpha for this sample was .87 .

Program Satisfaction Measure: Satisfaction with the program was measured via eight qualitative questions, answered anonymously, asking the participants to report on their experience of the group.

Please insert Table 3 about here (Correlation matrix) 
As seen in Table 4, used outcome measures displayed multicollinearity ranging from none to moderate suggesting that each instrument captured enough unique variance to merit its status as an outcome measure.

\section{Sample size}

To attain a power of 0.85 , with $\mathrm{p}<.05$ and an expected moderate effect size (Cohen's $d=0.5$ ), at least 75 participants should have been allocated to each condition (Rosnow, Rosenthal, \& Rubin, 2000), which was not possible given the pilot nature of the study and attainable resources. Thus, the study should be viewed as underpowered in this respect.

\section{Group Allocation}

Participants were randomly allocated to the ACT or TAU control groups using fixed lists with lines marked with ACT or TAU drawn from a random numbers table. In the same order the students gave their consent they were added to the fixed lists. The randomization was stratified for gender to help create one group for males. For the girls, a simple randomisation with a 1:1 allocation ratio was used. For the boys, a blocked randomisation was used. For the boys the randomisation ratio was 2:1 (two ACT participants per each TAU participant).

\section{Data Analyses}

Mixed Model Repeated Measures (MMRM) was used following the same method referred to previously in the Australian study. The only difference in the Swedish study was that school and age was not included as fixed effects.

\section{Results}

Table 2 shows the adjusted means for all outcome measures for the two conditions for each measurement occasion drawn from mixed model analyses. The MMRM results are shown in Table 2.

Tests of pre-differences were drawn from contrasts conducted MMRM analyses. At baseline the intervention group had marginally significantly higher stress [DASS-S, $(t(28)=-1.92 p=0.065$, Cohen's $d=$ 0.70], significantly higher depression [DASS-D, $t(28)=-2.84 p=0.008$, Cohen's $d=1.04$ ], and poorer general mental health [GHQ-12, $t(28)=-2.90, p=0.007$, Cohen's $d=1.06]$. No other measures were significantly 
different. All primary analyses were supplemented with analyses using baseline covariates to adjust for these differences.

Please insert Table 4 Mean, standard deviation, and MMRM about here about here

Please insert Figure 4. Primary outcome Sweden,

Intervention Effects on stress about here

Results for the Perceived Stress Scale (PSS) showed a significant effect for time $(p<.001)$, a nonsignificant effect for condition $(p=0.473)$, and a significant and large time by treatment interactions $(F(1,23.0)$ $=8.21, p=0.009$, Cohen's $d=1.20$ ). As is shown in Figure 4, the interaction was accounted for by the small and non-significant improvement in the TAU condition $($ Mdiff $=-2.29, \mathrm{SE}=1.44, t(22.99)=-1.59, p=0.125$, 95\% CI: $-5.27,0.69)$ and the large and significant improvement in the ACT condition (Mdiff $=-8.51, \mathrm{SE}=1.62$, $t(23.00)=-5.24, p<0.001,95 \% \mathrm{CI}:-11.87,-5.15)$. A similar analysis using the pre values of the scales with baseline differences (on the SWLS, DASS-S, and GHQ-12) showed the same significant results.

The secondary outcome variables were satisfaction with life as measured by Satisfaction with Life Scale (SWLS), Depression, Anxiety and Stress, as measured by the respective subscale in Depression (DASS-D), Anxiety (DASS-A) and Stress Scale (DASS-S), and General Health as measured by the General Health Questionnaire (GHQ-12). We will present the results without covariates but in all cases results that were significant were still so after a similar analysis using the pre values of the scales with baseline differences between conditions.

Results for the Satisfaction with Life Scale (SWLS) showed no significant results. Depression, Stress, and general mental health all showed only an effect for condition $(p<0.013,0.031$, and 0.03 , respectively). In these 
cases the baseline differences persisted into post but with no effect for time or the interaction with time and condition. Anxiety showed no effect for time $(p=0.983)$, or condition $(p=0.479)$ but did show a marginally significant and large interaction between time and condition $(F(1,24.038)=4.00, p=0.057$, Cohen's $d=0.80)$. The interaction was accounted for by the non-significant deterioration in the TAU condition $($ Mdiff $=0.95, \mathrm{SE}=$ $0.64, t(23.55)=1.48, p=0.15,95 \% \mathrm{CI}:-.376,2.277$.) and the non-significant improvement in the ACT condition $($ Mdiff $=-0.97, \mathrm{SE}=0.74, t(24.35)=-1.36, p=0.19,95 \% \mathrm{CI}:-2.45,0.50)$.

The primary process variable was considered to be mindfulness as measured by the Mindful Attention Awareness Scale (MAAS). Results for the MAAS showed a non-significant effect for time $(p=0.829)$, a nonsignificant effect for condition $(p=0.395)$, and a marginally significant and medium time by treatment interactions $(F(1,24.14)=3.68, p=0.067$, Cohen's $d=0.75$. As is shown in Table 2, the interaction was accounted for by the non-significant decrease of mindfulness skills in the TAU condition $($ Mdiff $=-3.92, \mathrm{SE}=$ $2.47, t(23.01)=-1.58, p=0.127,95 \% \mathrm{CI}:-9.04,1.20)$ and the non-significant improvements in the ACT condition $($ Mdiff $=3.12, \mathrm{SE}=2.71, t(24.90)=1.15, p=0.261,95 \% \mathrm{CI}:-2.47,8.71)$. Another process variable was psychological inflexibility, as measured by Avoidance and Fusion Questionnaire for Youth (AFQ-Y17). Results for the AFQ-Y17 showed no significant effects.

There were a total of 15 students (9 girls and 6 boys) randomized to the ACT intervention. At the end of the intervention 2 girls and 2 boys had dropped out of the groups ( 3 due to lack of time and 1 reported misfit of program). The remaining 11 students that received the ACT intervention participated on average 5.8 sessions (same for boys and girls) out of 8 sessions. The outcome of the intervention, as measured by the change in the quality of life and stress correlated significantly with course attendance in terms of number of attended sessions. Participants who attended a larger number of sessions also reported significantly higher quality of life (measured by SWLS, correlation $0.66, p=0.034$ ) and less depression, anxiety and stress (measured by DAS-S, correlation $0.62, p=0.042$ ). However, course attendance had no significant relationship to participants' report of mindfulness, psychological inflexibility, or general psychological health.

The majority of the participants (91\%) gave exclusively positive feedback on the course evaluation. Half of the participants felt that the course had been very valuable, and the rest evaluated it as quite valuable. All the respondents reported that they would recommend a friend to take this course. 


\section{Discussion}

These two studies examined the effects of a manualized brief targeted group intervention, based on Acceptance and Commitment Therapy, with adolescents in two different countries, Australia and Sweden. The ACT group format was compared to the usual care provided by schools.

The Australian study showed that on the primary outcome variable of depression (as measured by total score of RADS-2), there was greater improvement for those in the ACT group format, with a large effect size. RADS-2 measures current levels of depressive symptomatology along four dimensions (Dysphoric Mood, Anhedonia/Negative Affect, Negative Self-Evaluation and Somatic), effects in favour of the ACT intervention were found on all dimensions except the Somatic dimension. Effect sizes ranged from medium to large. The primary process variable was considered to be levels of psychological inflexibility (as measured by AFQ-Y8), and the short ACT group format was more effective than the usual care provided by schools in decreasing psychological inflexibility among the participants. The effect was of a medium size.

The Swedish study showed that on the primary outcome of stress (as measured by PSS), there was greater improvement for those in the ACT group format, with a large effect size. Regarding secondary outcomes the ACT group had a marginally significant decrease in anxiety as compared to usual care after the intervention, the effect was of a large size. The process variables were considered to be levels of psychological inflexibility (as measured by AFQ-Y17), and mindfulness (as measured by MAAS). There was a marginally significant increase in mindfulness skills in the ACT group compared to usual care with a medium effect size. Results for psychological inflexibility showed no significant effects.

Taken together, the ACT-intervention shows promise for adolescent participants who may be experiencing signs of depressive symptoms or high stress. In terms of student acceptability, both sites reported high rates of student acceptability, with almost unanimously positive qualitative reports and low dropout rates once they were in the program. The two facilitators of the Swedish ACT-interventions were new to ACT and had received a total of four days of training in ACT, suggesting that these methods can be disseminated into practical settings with reasonable training.

In the Swedish study the ACT-sessions were given after school hours, but as close to the last lesson as possible. Three out of the four students who dropped out of the ACT intervention gave "-Lack of time” as the reason for dropout. The majority of the ACT - participants (91\%) gave exclusively positive feedback on the 
written course evaluation. In the instances the participants gave a critique, it was about the fact that the intervention was after school hours. Delivering interventions as part of the regular curricula would avoid this problem and may be an approach worth investigation.

It is worth noting the increased mindfulness (marginally significant with medium effect size) for those in the ACT groups in the Swedish study. In the Swedish ACT-interventions there were no formal mindfulness exercises included during the sessions. Formal mindfulness exercises are commonly delivered (via CDs or files that are downloadable from the Internet) in ACT group interventions, but that was not done in the present study, suggesting that ACT exercises may share some of the same effects as are produced by formal mindfulness exercises.

In both studies, the ACT specific Avoidance and Fusion Questionnaire (AFQ-Y) was used as an outcome measure to assess psychological inflexibility (the opposite of psychological flexibility) in adolescents. The AFQ$\mathrm{Y}$ is designed to measure key areas of Acceptance and Commitment Therapy such as fusion and acceptance of negative thoughts and emotions. The Swedish study found no significant effects with this measure. This could be a problem with low power; a stronger replication with a larger sample size is needed to understand if it is a suitable process measure of change in the primary outcome measures.

These two studies showed that the targeted ACT intervention could increase adolescent's psychosocial health, a result that is congruent with previous findings on school based interventions for depression where targeted interventions has been found to be the most effective, with effect sizes for all programs ranging from 0.21 to 1.40 (Calear \& Christensen, 2010; Horowitz et al., 2007; Merry et al., 2011; Merry et al., 2004). To our knowledge, however, the impact of a school based ACT intervention for stress has not been examined, studies are underway, but this is the first one published. Our results are congruent with previous positive findings on short ACT group interventions for stress among adults (Bethay, Wilson, Schnetzer, Nassar, \& Bordieri, 2013; Bond \& Bunce, 2000; Brinkborg et al., 2011; Flaxman \& Bond, 2010a, 2010b).

Taken together, the ACT-intervention seems to be a promising intervention for reducing stress and depressive symptoms among young adolescents in school and should be tested in full-sized studies. This intervention also has a firm theoretical basis and potential to work trans diagnostically. Because of the cost of depression and stress to society, and its relationship to suicide attempts and completed suicide, this is an important public health issue (Merry et al., 2011). Since this is a fairly time-limited intervention, and in Sweden the facilitators received only four days of training in ACT, this might indicate that this intervention can be a promising program suitable for a larger roll-out. If this ACT group treatment is proven effective in full-sized 
studies it could have a profound effect on future service provision for adolescent mental health problems. It would be especially helpful for the large group of adolescents that currently can't expect help from the Child and Youth psychiatry or Outpatient psychiatry because their problems are not "acute or severe enough".

\section{Limitations of the present study}

Although results of the present study appear promising, it is important to interpret the findings in light of limitations. Firstly, the findings are limited without long-term follow-up. These studies were pilot programs, aimed at testing the acceptability of ACT for high school students, however; as many school interventions have not retained their positive effects on the outcome measures over time, long term follow-up is needed to confirm the findings. Second, the sample size of the present study was relatively small, and of a limited age range, making it hard to generalise results to the general population and giving the statistical analysis low power. Third, most participants were girls limiting the interpretation of results to this gender. Fourth, the Swedish school where the study was conducted differed from other Swedish regular public high schools in two respects: a) All the students at this school qualified to study at upper secondary school at the age of 15 compared to $89 \%$, which is the national average rate, and b) Parent's income level was twice as high as the Swedish family mean. Fifth, participants in Australia and Sweden were all volunteers. This is true for participants in both the ACT interventions and in treatment as usual, and the participants were randomized (except boys in Australia) between the groups so the observed effects seem to be stable. Nevertheless, the fact that the students volunteered might limit generalizability since we do not know if the students that volunteered differ from the students that did not volunteer for the study. Sixth, in the Australian study there was a significant difference before treatment between the ACT- and intervention groups regarding levels of depression (as measured by RADS-2) that was the primary outcome. An alternative interpretation of the results could be that the ACT-intervention group was regressing towards the mean. Seventh, the ACT intervention differs from treatment as usual in two important ways, a) the ACT groups got more treatment time on average then the students that received individual support from the school health care, and b) in the ACT intervention the participants met in group format (as opposed to individual treatment by school health care) and we do not know what role possible peer support or meeting peers in a similar situation played. Eight, the ACT intervention consists of eight sessions that usually is delivered over a time period of 8-10 weeks. Due to school time scheduling the eight sessions in Sweden were squeezed into a time period of six weeks, which may have affected the participants' ability to benefit from the the course 
optimally. Ninth, it may be worth noting that two slightly different versions of the Avoidance and Fusion Questionnaire were used to measure the primary process variable of psychological inflexibility, in the Australian study the short form of 8 items was used, and in the Swedish study the full form with 17 items was used. Finally, the data were all self-report questionnaires, a future study should include additional objective measures, perhaps school attendance or grades.

\section{Future research}

Future research would benefit from having a more rigorous design that in more detail can help the theoretical advancement of interventions by designing studies that can examine putative processes of change by examining possible moderators and mediators of effects. Future studies should also consider measuring therapist adherence and competency in delivering the intervention. Those two studies were conducted in real life settings (effectiveness studies), a recommendation is to also conduct future studies as effectiveness studies. Future studies would benefit from larger sample sizes. If large enough sample sizes can be attained it would be of great help to test this or similar interventions as universal prevention. Given the practical difficulties inherent in implementing a targeted program, pursuing the implementation of universal depression prevention programs is warranted. In prevention programs it is often the asymptomatic group that yield more cases than the subsyndromal group (Merry et al., 2011). Future research can even further address the gap that still remains concerning possible placebo effect through comparing the promising programs to attention control or credible alternatives in effectiveness trials. In future research it will be important to measure not only reduction in depression or stress, but also levels of youth functioning in life, life quality, and the on-going impact into the medium and long term. Finally future studies should include additional objective follow-up measures, perhaps school attendance or grades with the aim of increasing objectivity in the outcome measures as well as being able to conduct relevant long term follow-ups, preferably, at least 12 months and longer.

\section{Conflict of interest:}

The authors declare that they have no conflict of interest. 


\section{References}

Bethay, J. S., Wilson, K. G., Schnetzer, L. W., Nassar, S. L., \& Bordieri, M. J. (2013). A controlled pilot evaluation of acceptance and commitment training for intellectual disability staff. Mindfulness, 4(2), 113-121. doi:10.1007/s12671-012-0103-8

Biglan, A., Hayes, S. C., \& Pistorello, J. (2008). Acceptance and commitment: Implications for prevention science. Prevention Science, 9(3), 139-152. doi:10.1007/s11121-008-0099-4

Bond, F. W., \& Bunce, D. (2000). Mediators of change in emotion-focused and problem-focused worksite stress management interventions. Journal of Occupational Health Psychology, 5(1), 156-163. doi:10.1037//1076-8998.5.1.156

Brinkborg, H., Michanek, J., Hesser, H., \& Berglund, G. (2011). Acceptance and commitment therapy for the treatment of stress among social workers: A randomized controlled trial. Behaviour Research and Therapy, 49(6-7), 389-398. doi:10.1016/j.brat.2011.03.009

Brown, K. W., \& Ryan, R. M. (2003). The benefits of being present: Mindfulness and its role in psychological well-being. Journal of Personality and Social Psychology, 84(4), 822-848. doi:10.1037/00223514.84 .4 .822

Brown, T. A., Chorpita, B. F., Korotitsch, W., \& Barlow, D. H. (1997). Psychometric properties of the Depression Anxiety Stress Scales (DASS) in clinical samples. Behaviour Research and Therapy, 35(1), 79-89. doi:10.1016/s0005-7967(96)00068-x

Calear, A. L., \& Christensen, H. (2010). Systematic review of school-based prevention and early intervention programs for depression. Journal of Adolescence, 33(3), 429-438. doi:10.1016/j.adolescence.2009.07.004

Campbell, A., Walker, J., \& Farrell, G. (2003). Confirmatory factor analysis of the GHQ-12: Can I see that again? Australian \& New Zealand Journal of Psychiatry, 37(4), 475-483. doi:10.1046/j.14401614.2003.01208.x

Cohen, J. (1988). Statistical power analysis for the behavioral sciences (2nd ed.). Hillsdale, NJ: Erlbaum.

Cohen, S., \& Janicki-Deverts, D. (2012). Who's stressed? Distributions of psychological stress in the United States in probability samples from 1983, 2006, and 2009. Journal of Applied Social Psychology, 42(6), 1320-1334. doi:10.1111/j.1559-1816.2012.00900.x

Cohen, S., Kessler, R. C., \& Underwood Gordon, L. (1995). Measuring stress: A guide for health and social scientists. New York, NY: Oxford University Press. 
Duckworth, A. L., Kim, B., \& Tsukayama, E. (2013). Life stress impairs self-control in early adolescence. Frontiers in Psychology, 3, 608. doi:10.3389/fpsyg.2012.00608

Eskin, M., \& Parr, D. (1996). Introducing a Swedish version of an instrument measuring mental stress (Reports from the Department of Psychology, No. 813). Stockholm, Sweden: Stockholm University

Flaxman, P. E., \& Bond, F. W. (2010a). A randomised worksite comparison of acceptance and commitment therapy and stress inoculation training. Behaviour Research and Therapy, 48(8), 816-820. doi:10.1016/j.brat.2010.05.004

Flaxman, P. E., \& Bond, F. W. (2010b). Worksite stress management training: Moderated effects and clinical significance. Journal of Occupational Health Psychology, 15(4), 347-358. doi:10.1037/a0020522

Flay, B. R., Biglan, A., Boruch, R. F., Castro, F. G., Gottfredson, D., Kellam, S., Mościcki, E., K., Schinke, S., Valentine, J., C. \& Ji, P. (2005). Standards of evidence: Criteria for efficacy, effectiveness and dissemination. Prevention Science, 6(3), 151-175. doi:10.1007/s11121-005-5553-y

Gao, F., Luo, N., Thumboo, J., Fones, C., Li, SC., \& Cheung, YB. (2004). Does the 12-item General Health Questionnaire contain multiple factors and do we need them? Health and Quality of Life Outcomes, 2, 63. doi:10.1186/1477-7525-2-63

Goodman, R. (2001). Psychometric properties of the Strengths and Difficulties Questionnaire. Journal of the American Academy of Child and Adolescent Psychiatry, 40(11), 1337-1345. doi:10.1097/00004583200111000-00015

Goodman, R., Meltzer, H., \& Bailey, V. (2003). The Strengths and Difficulties Questionnaire: A pilot study on the validity of the self-report version. International Review of Psychiatry, 15(1-2), 173-177. doi:10.1080/0954026021000046137

Grant, K. E., Compas, B. E., Thurm, A. E., McMahon, S. D., \& Gipson, P. Y. (2004). Stressors and child and adolescent psychopathology: Measurement issues and prospective effects. Journal of Clinical Child \& Adolescent Psychology, 33(2), 412-425. doi:10.1207/s15374424jccp3302_23

Greco, L. A., Baer, R. A., \& Lambert, W. (2008). Psychological inflexibility in childhood and adolescence: Development and evaluation of the Avoidance and Fusion Questionnaire for Youth. Psychological Assessment, 20(2), 93-102. doi: 10.1037/1040-3590.20.2.93

Hankin, B. L., Mermelstein, R., \& Roesch, L. (2007). Sex differences in adolescent depression: Stress exposure and reactivity models. Child Development, 78(1), 279-295. doi:10.1111/j.1467-8624.2007.00997.x 
Hayes, L. L., Boyd, C. P., \& Sewell, J. (2011). Acceptance and commitment therapy for the treatment of adolescent depression: A pilot study in a psychiatric outpatient setting. Mindfulness, 2(2), 86-94. doi:10.1007/s12671-011-0046-5

Hayes, L. L., \& Rowse, J. (2008). Acceptance and commitment therapy: Experiential adolescent group. Retrieved from Association for Contextual Behavioral Science website: http://contextualpsychology.org/treatment_protocols

Hayes, S. C., Luoma, J. B., Bond, F. W., Masuda, A., \& Lillis, J. (2006). Acceptance and commitment therapy: Model, processes and outcomes. Behaviour Research and Therapy, 44(1), 1-25. doi:10.1016/j.brat.2005.06.006

Hayes, S. C., \& Strosahl, K. D. (Eds.). (2004). A practical guide to acceptance and commitment therapy. New York, NY: Springer-Verlag.

Hayes, S. C., Strosahl, K. D., \& Wilson, K. G. (2012). Acceptance and commitment therapy: The process and practice of mindful change (2nd ed.). New York, NY: Guilford Press.

Heffner, M., Sperry, J., Eifert, G. H., \& Detweiler, M. (2002). Acceptance and commitment therapy in the treatment of an adolescent female with anorexia nervosa: A case example. Cognitive and Behavioral Practice, 9(3), 232-236. doi:10.1016/s1077-7229(02)80053-0

Hewitt, P. L., Flett, G. L., \& Mosher, S. W. (1992). The Perceived Stress Scale: Factor structure and relation to depression symptoms in a psychiatric sample. Journal of Psychopathology and Behavioral Assessment, 14(3), 247-257. doi:10.1007/BF00962631

Horowitz, J. L., Garber, J., Ciesla, J. A., Young, J. F., \& Mufson, L. (2007). Prevention of depressive symptoms in adolescents: A randomized trial of cognitive-behavioral and interpersonal prevention programs. Journal of Consulting and Clinical Psychology, 75(5), 693-706. doi:10.1037/0022-006x.75.5.693

Jakobsson, C., \& Wellin, J. (2006). Acceptance and commitment therapy and stress in school - A two year follow up of a randomized controlled trial (Master's thesis, Uppsala University, Sweden). Retrieved from http://www.livskompass.se/wp-content/uploads/2012/11/act-stress-i-skolan-2arsuppfoljning.pdf

Keenan-Miller, D., Hammen, C. L., \& Brennan, P. A. (2007). Health outcomes related to early adolescent depression. Journal of Adolescent Health, 41(3), 256-262. doi:10.1016/j.jadohealth.2007.03.015

Livheim, F. (2004). Acceptance and commitment therapy in school setting - To cope with stress, a randomized controlled trial (Master's thesis, Uppsala University, Sweden). Retrieved from http://www.livskompass.se/wp-content/uploads/2012/11/act-i-skolan-2004.pdf 
Merry, S. N., Hetrick, S. E., Cox, G. R., Brudevold-Iversen, T., Bir, J. J., \& McDowell, H. (2011). Psychological and educational interventions for preventing depression in children and adolescents. Cochrane Database of Systematic Reviews, 2011(12), Art. No.: CD003380. doi:10.1002/14651858.CD003380.pub3

Merry, S. N., McDowell, H., Hetrick, S., Bir, J., \& Muller, N. (2004). Psychological and/or educational interventions for the prevention of depression in children and adolescents. Cochrane Database of Systematic Reviews, Art. No.: CD003380. doi:10.1002/14651858.CD003380.pub2

Merry, S. N., \& Spence, S. H. (2007). Attempting to prevent depression in youth: A systematic review of the evidence. Early Intervention in Psychiatry, 1(2), 128-137. doi:10.1111/j.1751-7893.2007.00030.x

Metzler, C. W., Biglan, A., Noell, J., Ary, D. V., \& Ochs, L. (2000). A randomized controlled trial of a behavioral intervention to reduce high-risk sexual behavior among adolescents in STD clinics. Behavior Therapy, 31(1), 27-54. doi:10.1016/s0005-7894(00)80003-9

Montazeri, A., Hole, D. J., Milroy, R., McEwen, J., \& Gillis, C. R. (2004). Does knowledge of cancer diagnosis affect quality of life? A methodological challenge. BMC Cancer, 4(21). doi:10.1186/1471-2407-4-21

Mrazek, P. J., \& Haggerty, R. J. (1994). Reducing risks for mental disorders: Frontiers for preventive intervention research. Washington, DC: National Academies Press.

Muris, P., Meesters, C., Eijkelenboom, A., \& Vincken, M. (2004). The self-report version of the Strengths and Difficulties Questionnaire: Its psychometric properties in 8- to 13-year-old non-clinical children. British Journal of Clinical Psychology, 43(4), 437-448. doi:10.1348/0144665042388982

Neto, F. (1993). The Satisfaction With Life Scale: Psychometic properties in an adolescent sample. Journal of Youth and Adolescence, 22(2), 125-134. doi:10.1007/BF01536648

Ost, L.-G. (2008). Efficacy of the third wave of behavioral therapies: A systematic review and meta-analysis. Behaviour Research and Therapy, 46(3), 296-321. doi:10.1016/j.brat.2007.12.005

Parker, G., \& Roy, K. (2001). Adolescent depression: A review. Australian and New Zealand Journal of Psychiatry, 35(5), 572-580. doi:10.1046/j.1440-1614.2001.00934.x

Pavot, W., \& Diener, E. (1993). Review of the Satisfaction With Life Scale. Psychological Assessment, 5(2), 164-172. doi:10.1037/1040-3590.5.2.164

Politi, P. L., Piccinelli, M., \& Wilkinson, G. (1994). Reliability, validity and factor structure of the 12 -item General Health Questionnaire among young males in Italy. Acta Psychiatrica Scandinavica, 90(6), 432437. doi:10.1111/j.1600-0447.1994.tb01620.x 
Powers, M. B., Vording, M. B. Z. V. S., \& Emmelkamp, P. M. G. (2009). Acceptance and commitment therapy: A meta-analytic review. Psychotherapy and Psychosomatics, 78(2), 73-80. doi:10.1159/000190790

Rao, U., Hammen, C., Ortiz, L. R., Chen, L. A., \& Poland, R. E. (2008). Effects of early and recent adverse experiences on adrenal response to psychosocial stress in depressed adolescents. Biological Psychiatry, 64(6), 521-526. doi:10.1016/j.biopsych.2008.05.012

Reynolds, W. M. (2002). Reynold's Adolescent Depression Scale-2nd Edition: Professional manual. Lutz, FL.: Psychological Assessment Resources.

Reynolds, W. M. (2010). Reynolds Adolescent Depression Scale. In I. B. Weiner \& W. E. Craighead (Eds.), The Corsini Encyclopedia of Psychology. Hoboken, NJ: John Wiley \& Sons

Rosenthal, R., \& Rosnow, R. L. (1991). Essentials of behavioral research: Methods and data analysis (2nd ed.): New York, NY: McGraw-Hill.

Rosnow, R. L., Rosenthal, R., \& Rubin, D. B. (2000). Contrasts and correlations in effect-size estimation. Psychological Science, 11(6), 446-453. doi: 10.1111/1467-9280.00287

Statistics Sweden. (2010). Undersökningarna av barns levnadsförhållanden [The surveys of children's living conditions]. Pressrelease from SCB 16/08/10. No. 2010:209: Retrieved from Statistics Sweden: http://www.scb.se/Pages/PressRelease_298563.aspx

Theodore-Oklota, C., \& Orsillo, S. (2011). A mindfulness-based program aimed at reducing the impact of relational aggression. Paper presented at 2011 Association for Contextual Behavioral Science Annual World Conference 9, Parma, Italy.

Verbeke, G., \& Molenberghs, G. (2000). Linear mixed models for longitudinal data. New York, NY: SpringerVerlag.

Wackerly, D., Mendenhall, W., \& Scheaffer, R. L. (2008). Mathematical statistics with applications (7th ed.). Belmont, CA: Thomson Learning.

Wicksell, R. K., Melin, L., Lekander, M., \& Olsson, G. L. (2009). Evaluating the effectiveness of exposure and acceptance strategies to improve functioning and quality of life in longstanding pediatric pain. A randomized controlled trial. Pain, 141(3), 248-257. doi:10.1016/j.pain.2008.11.006

Wilson, K. G., \& Murrell, A. R. (2002). ACT for LIFE (Lifting Individuals for Future Endeavors): A treatment manual for at-risk youth. Retrieved from Association for Contextual Behavioral Sciences website: http://contextualscience.org/files/ACT_for_Life_Protocol_2_12_03\%5B1\%5D_0.pdf 
World Health Organization. (2011). Young people: Health risks and solutions. (Fact sheet No. 345). Retrieved from World Health Organization website:

http://www.who.int/mediacentre/factsheets/fs345/en/index.html.

World Medical Association. (2008). Declaration of Helsinki: Ethical principle for medical research involving human subjects. Retrieved from http://www.healthscience.net/resources/declaration-of-helsinki/

Figure 1. Participant Flow Diagram Australia

\section{Australia}

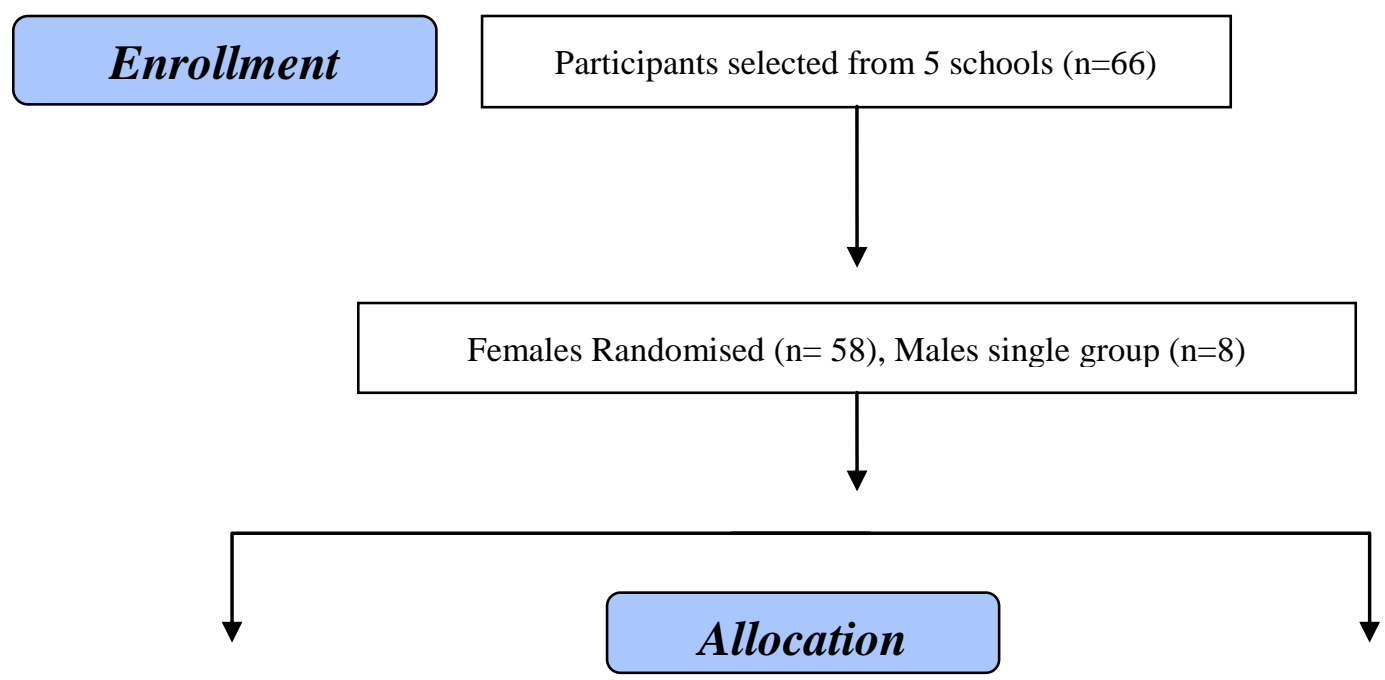

Allocated to ACT intervention $(\mathrm{n}=32$ females, 8 males)

Received allocated intervention $(n=35)$

Did not receive intervention:

Reason unknown $n=3$, Left school $n=2$
Allocated to treatment as usual, TAU ( $\mathrm{n}=26$ females)

Received allocated intervention $(n=20)$

Did not receive intervention (given reasons):

Reason unknown $n=3$, Left school $n=3$,
Analysed $(n=32)$

Incomplete post data $(n=3)$
Analysed ( $\mathrm{n}=19)$

Incomplete post data $(\mathrm{n}=1)$ 
Figure 2. Primary outcome Australia, Intervention Effects on depression

\section{Depressive symptoms \\ (RADS- 2)}

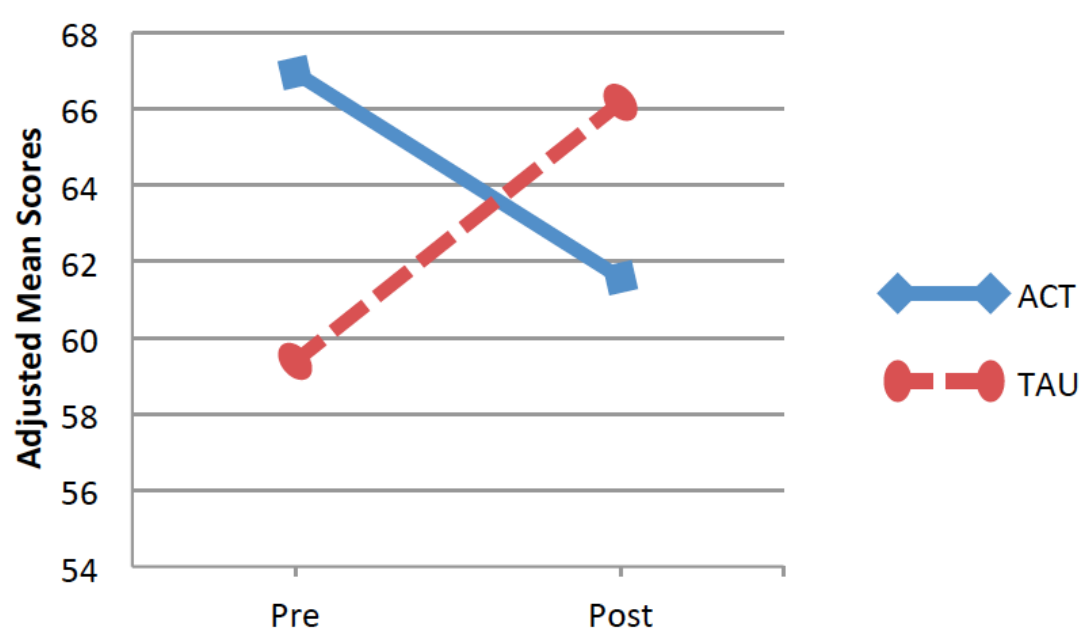

Results for the Reynolds Adolescent Depression Scale-2 (RADS-2). Analysis of covariance using school and age as covariate. 
Figure 3. Participant Flow Diagram Sweden

\section{Sweden}

Assessed for eligibility via screening $(n=247)$

\section{Enrollment}
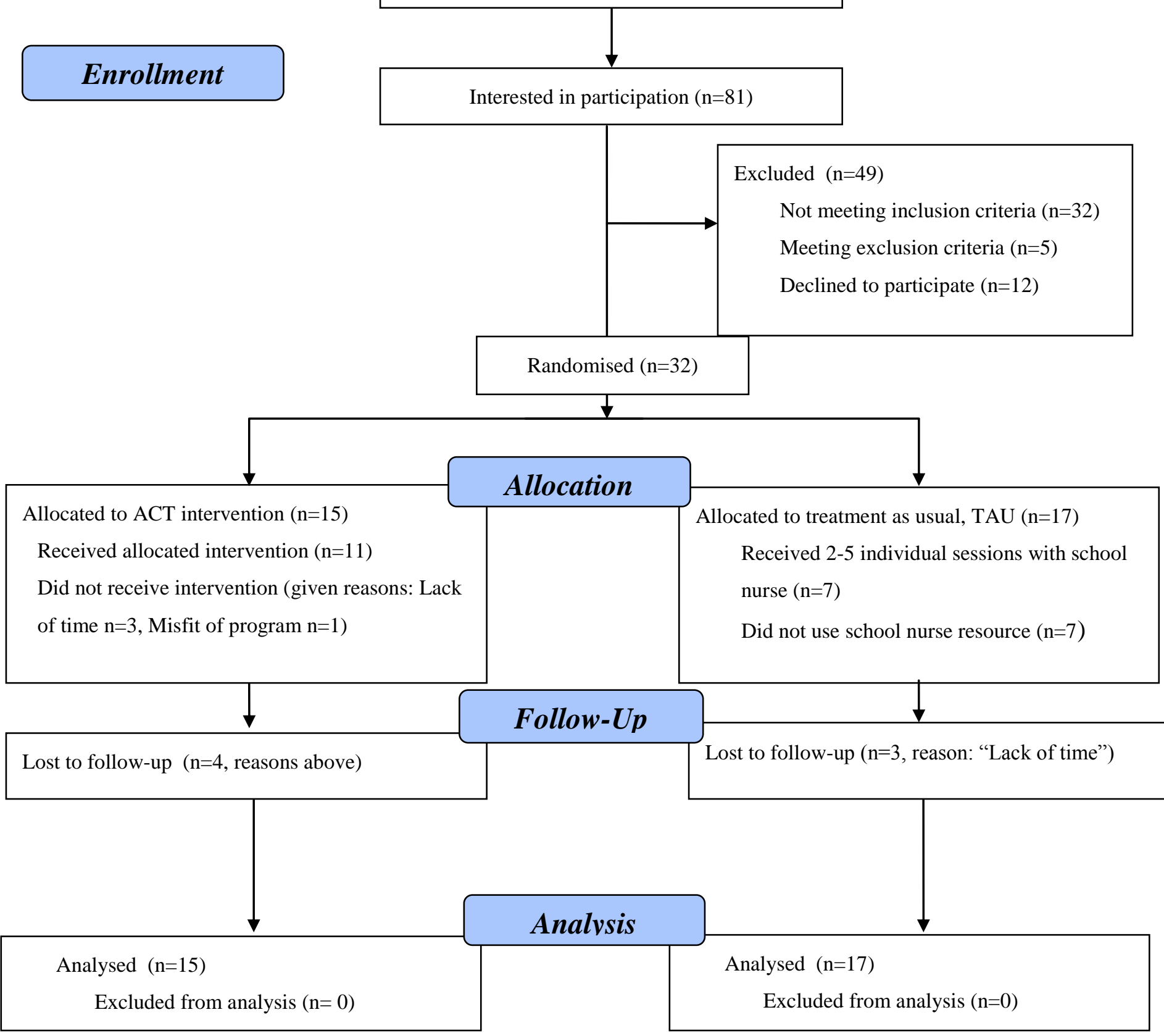
Figure 4. Primary outcome Sweden, Intervention Effects on stress

\section{Stress}

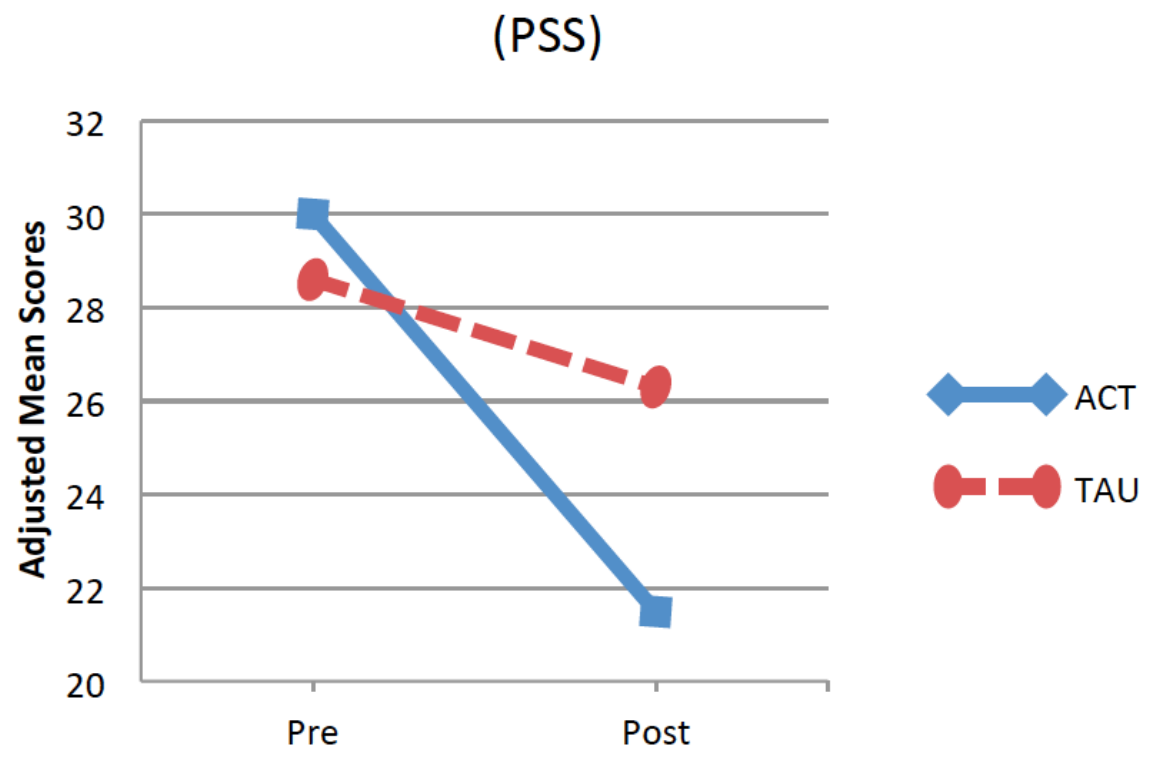

Results for The Perceived Stress Scale (PSS).

For Swedish adults, low levels of stress is defined as 24 points or less, and high levels of stress defined as 25 points or more (Eskin \& Parr, 1996).

\section{Table 1.}

Summary of Intercorrelations for Scores on the RADS-2 Total Score, RADS-s Subscales, and AFQ-Y8

\begin{tabular}{|c|c|c|c|c|c|c|}
\hline Measure & $R A D S-2$ & Dys & Neg. Affect & Neg Self Eva. & Somatic & $A Y Q-Y 8$ \\
\hline Total RADS-2 & - & & & & & \\
\hline Dysphoria & $.91 * *$ & - & & & & \\
\hline Neg. Affect & $.76 * *$ & $.60 * *$ & - & & & \\
\hline Neg. Self Ev & $.93 * *$ & $.79 * *$ & $.65^{* *}$ & - & & \\
\hline
\end{tabular}


Somatic

$.86 * *$

$.76^{* *}$

$.50 * *$

$.73 * *$

AFQ-Y8

Pearson Correlation (2-tailed) for the Australian participants' $(n=63)$ pre-intervention scores. RADS-2 = Reynolds' Adolescent Depression; RADS-2 Subscales - Neg. Affect $=$ Anhedonia/Negative Affect, Neg Self Ev $=$ Negative Self Evaluation, Somatic $=$ Somatic Complaints; $A F Q-Y 8=$ Avoidance and Fusion QuestionnaireYouth 8 items

$* * p<.01$

$* p<.05$ 
Table 2.

Australian study - Mean, standard deviation, and Mixed Model Repeated Measures (MMRM) for the intervention versus the control group. Since the group of boys were not randomized, output for the girls only are also reported. The girls were fully randomized. All results presented are intent-to-treat analyses.

MMRM

ACT Control $\quad F$ statistic, $p$-values,

effect sizes in Cohen's $d$

\begin{tabular}{|c|c|c|c|c|c|c|c|}
\hline Measures & $\begin{array}{c}\text { Pre } \\
M(S E)\end{array}$ & $\begin{array}{c}\text { Post } \\
M(S E)\end{array}$ & $\begin{array}{c}\text { Pre } \\
M(S E)\end{array}$ & $\begin{array}{r}\text { Post } \\
M(S E)\end{array}$ & $\begin{array}{c}\text { F } \\
\text { statistic }\end{array}$ & $\begin{array}{c}p- \\
\text { value }\end{array}$ & $\begin{array}{l}\text { Cohen's } \\
\qquad d\end{array}$ \\
\hline \multicolumn{8}{|l|}{ Reynolds Adolescent } \\
\hline Depression Scale & & & & & & & \\
\hline $\begin{array}{l}\text { Total RADS-2 } \\
\text { (everyone) }\end{array}$ & 66.95 (2.72) & $61.57(3.80)$ & $58.54(3.62)$ & $65.48(5.13)$ & 7.59 & 0.008 & $0.86 * *$ \\
\hline $\begin{array}{l}\text { Total RADS-2 } \\
\text { (girls only) }\end{array}$ & $69.95(2.85)$ & $64.95(4.17)$ & $59.38(3.37)$ & $66.17(5.05)$ & 5.76 & 0.021 & $0.78^{*}$ \\
\hline Dysphoria (everyone) & $19.73(0.86)$ & $17.67(1.18)$ & $15.91(1.15)$ & $18.08(1.61)$ & 6.01 & 0.017 & $0.77^{*}$ \\
\hline Dysphoria (girls only) & $20.70(0.89)$ & $18.75(1.30)$ & $16.31(1.06)$ & $18.46(1.60)$ & 4.74 & 0.036 & $0.70^{*}$ \\
\hline Neg. Affect (everyone) & $12.63(0.65)$ & $11.31(0.70)$ & $11.56(0.87)$ & $13.09(0.96)$ & 8.31 & 0.006 & $0.89 * *$ \\
\hline Neg. Affect (girls only) & $13.07(0.74)$ & $11.60(0.80)$ & $11.65(0.87)$ & $13.21(0.97)$ & 7.85 & 0.008 & $0.90 * *$ \\
\hline Neg Self Ev.(everyone) & $16.60(0.90)$ & $15.43(1.22)$ & $14.31(1.20)$ & $16.58(1.66)$ & 4.63 & 0.037 & $0.67^{*}$ \\
\hline Neg Self Ev.(girls only) & $17.26(1.00)$ & $16.66(1.31)$ & $14.42(1.18)$ & $16.73(1.60)$ & 2.81 & 0.101 & 0.54 \\
\hline Somatic (everyone) & $18.03(0.80)$ & $17.09(1.03)$ & $16.65(1.06)$ & $17.62(1.39)$ & 2.62 & 0.113 & 0.50 \\
\hline Somatic (girls only) & $18.99(0.78)$ & $17.91(1.12)$ & $16.87(0.92)$ & $17.83(1.36)$ & 2.51 & 0.122 & 0.51 \\
\hline AFQ-Y8 (everyone) & $12.06(1.23)$ & $10.23(1.24)$ & $9.13(1.62)$ & $11.21(1.62)$ & 5.74 & 0.021 & $0.73^{*}$ \\
\hline AFQ-Y8 (girls only) & $13.57(1.34)$ & $11.41(1.33)$ & $9.39(1.57)$ & $11.53(1.55)$ & 5.77 & 0.021 & $0.76^{*}$ \\
\hline
\end{tabular}

Note: Analysis of covariance using school and age as covariate 
$M$ means, $S E$ standard error (everyone, $N=51$, girls only, $N=32$ )

Neg. Affect Anhedonia/Negative Affect, Neg Self Ev Negative Self Evaluation, Somatic Somatic Complaints AFQ-Y8 Avoidance and Fusion Questionnaire-Youth 8 items

Cutoffs suggested by Cohen: small $>.20$, medium $>.50$, large $>.80$

$* p<.05 . * * p<.01$

For analyses with probabilities of $p=<0.15$ effect sizes were calculated. Note that "golden standard" for interpreting effect sizes usually is set at $p=<0.05$ 
Table 3.

Summary of Intercorrelations for Scores on the PSS, MAAS, DAS-S 21, AFQ-Y17, GHQ-12, and SWLS

\begin{tabular}{|c|c|c|c|c|c|c|}
\hline Measure & $P S S$ & $M A A S$ & $S W L S$ & $D A S-S 21$ & $A F Q-Y 17$ & $G H Q-12$ \\
\hline$P S S$ & - & & & & & \\
\hline$M A A S$ & $-.54 * *$ & - & & & & \\
\hline$S W L S$ & $-.37 *$ & .09 & - & & & \\
\hline$D A S-S 21$ & $.58 * *$ & $-.47 * *$ & $-.59 * *$ & - & & \\
\hline$A F Q-Y 17$ & $.40 *$ & -.27 & $-.67 * *$ & $.73 * *$ & - & \\
\hline$G H Q-12$ & $.54 * *$ & -.27 & $-.71 * *$ & $.72 * *$ & $.73 * *$ & - \\
\hline
\end{tabular}

Pearson Correlation (2-tailed) for the Swedish participants' $(n=30)$ pre-intervention scores. PSS $=$ Perceived Stress Scale; MAAS = Mindful Attention Awareness Scale; SWLS = Satisfaction with Life Scale; DAS-S $21=$ Depression, Anxiety and Stress Scale; AFQ-Y17 = Avoidance and Fusion Questionnaire for Youth and GHQ-12 $=$ General Health Questionnaire

$* * p<.01$

$* \quad p<.05$ 


\section{Table 4.}

Swedish study - Mean, standard deviation, and Mixed Model Repeated Measures (MMRM) for the intervention versus the control group. All results presented are intent-to-treat analyses.

\section{MMRM}

ACT Control $\quad F$ statistic, $p$-values and effect sizes in Cohen's $d$

\begin{tabular}{|c|c|c|c|c|c|c|c|}
\hline & Pre & Post & Pre & Post & $F$ & $p-$ & Cohen's \\
\hline Measures & $M(S E)$ & $M(S E)$ & $M(S E)$ & $M(S E)$ & statistic & value & $d$ \\
\hline Stress: PSS & $30.00(1.49)$ & $21.49(2.18)$ & $28.60(1.49)$ & $26.30(2.04)$ & 8.21 & 0.009 & $1.20 * *$ \\
\hline Mindfulness: MAAS & $59.00(3.04)$ & $62.12(2.98)$ & $59.27(3.04)$ & $55.35(2.77)$ & 3.68 & 0.067 & 0.75 \\
\hline Anxiety: DAS S & $6.13(0.95)$ & $5.16(0.99)$ & $4.27(0.95)$ & $5.22(0.94)$ & 4.00 & 0.057 & 0.80 \\
\hline Depression: DAS S & $7.80(0.85)$ & $7.98(1.24)$ & $4.40(0.85)$ & $4.18(1.17)$ & 0.11 & 0.742 & - \\
\hline Stress: DAS S & $10.40(1.08)$ & $10.26(0.93)$ & $7.47(1.08)$ & $7.37(0.87)$ & 0.00 & 0.971 & - \\
\hline GHQ - 12 & $16.87(1.35)$ & $13.62(1.53)$ & $11.33(1.35)$ & $11.19(1.40)$ & 2.47 & 0.130 & - \\
\hline SWLS & $21.93(1.27)$ & $23.49(1.36)$ & $24.53(1.27)$ & $23.96(1.30)$ & 2.52 & 0.126 & - \\
\hline AFQ-Y17 & $26.40(2.74)$ & $23.37(3.01)$ & $20.33(2.74)$ & $19.32(2.80)$ & 0.35 & 0.562 & - \\
\hline
\end{tabular}

$M$ means, $S E$ standard error (ACT, $N=11$, Control, $N=14$ )

PSS The Perceived Stress Scale, MAAS Mindful Attention Awareness Scale, DAS S Depression, Anxiety and Stress Scale, GHQ-12 General Health Questionnaire, SWLS Satisfaction with Life Scale, $A F Q-Y 17$ Avoidance and Fusion Questionnaire-Youth 17 items.

Cutoffs suggested by Cohen: small $>.20$, medium $>.50$, large $>.80$ $* p<.05 . * * p<.01$.

Analyses with probabilities of $p=<0.10$ but $>0.05$ were referred to as "marginally significant" and were interpreted. 